\title{
The Informational Basis of Social Judgments: Memory for Integrated and Nonintegrated Trait Descriptions
}

\author{
Eugene Burnstein and YaAcov Schul \\ University of Michigan
}

Received January 9, 1981

\begin{abstract}
The representation of trait descriptions in memory depends not only on the properties of this information (e.g., whether it is consistent) but also on the operations performed on it (e.g., whether it has been integrated). If a set of traits merely has to be comprehended without being integrated (called a discrete judgment), then an inconsistent set is processed just as readily as a consistent one. However, if integration is required, that is, a coherent impression must be formed (called an integrative judgment), consistent trait descriptions are processed more rapidly than inconsistent ones. The effect of such differences in processing on the representation was shown in recognition memory performance: First, following integrative judgment a trait description was more accessible than following a discrete judgment. Second, under integrative judgment, memory for a consistent trait description was no greater than that for an inconsistent description, whereas under discrete judgment, memory for the former was superior to that for the latter. This suggests that when an impression is formed, namely, under integrative judgment, the traits describing a person are associated with each other via prior knowledge, and thus are represented as a unified structure; otherwise each trait is stored as a separate piece of information.
\end{abstract}

The present study is concerned with two related issues in opinion formation: If there is an inconsistency between arguments in a message (i) when is the inconsistency resolved and (ii) how does the resolution of the inconsistency affect the representation of this information in memory? Earlier (Burnstein \& Schul, 1982) we described several basic operations performed during opinion formation, namely, recognition (initial encoding), comprehension (elaborative encoding), unification of the encoded information (integration), and finally, an evaluation in light of this integrated knowledge (decision). In the course of this analysis it was

The research reported here was supported by a grant from the National Science Foundation (BNS 7915416). We thank Paula Diamond for her help in designing and carrying out the study. Address reprint requests to Eugene Burnstein. Research Center for Group Dynamics, Institute for Social Research, University of Michigan, Ann Arbor, MI 48106. 
suggested that a consistency check would not be performed automatically every time two or more arguments are processed. Rather, it should come into play when this information has to be integrated. ${ }^{1}$ At this point in opinion formation the arguments have to be interpreted in unified fashion and inconsistencies must somehow be resolved. A consistency check, therefore, is only one of several distinct operations performed between the initial encoding and the decision. If this is the case, differences in consistency should have pronounced effects at those points in opinion formation that involve integration but little or no effects otherwise.

To underline the difference between opinion formation processes that include integration from those that do not, let us call the former an integrative judgment, and the latter, a discrete judgment. Our experiment, therefore, examines (i) the processing of consistent and inconsistent trait descriptions and (ii) the representation of these descriptions in memory following either discrete judgment or integrative judgment. Recall that when trait descriptions are inconsistent an integrative judgment requires that the inconsistencies be resolved. This means that a schema must be found that provides a sensible, coherent interpretation of what, at first glance, seems to be incompatible traits. By definition such a schema is not the typical or dominant one in this contextotherwise the description would have at once been perceived as consistent-and thus, it is relatively inaccessible (Holmes, 1979; Smith, Shoben, \& Rips, 1974). As a result, an integrative judgment based on inconsistent trait information ought to take longer than one based on consistent trait information.

Discrete judgments encode each piece of information separately; they are typically performed in assessing frequency (e.g., "Did the group have more than three members named Igor?"), co-occurrence (e.g., "Did the group have both male and female members?"), or a common feature (e.g., "Were the group members all bald?"). Such operations do not call for an integrating schema; instead they can be carried out simply by making a separate estimate for each piece of information and keeping a tally of the results. Hence, there is no need either to check the information for inconsistencies or to reconcile those that happen to be detected. It follows, then, that the time needed to make a discrete judgment should be invariant with the consistency of the trait description.

According to our analysis, the representation of trait information will also differ as a function of the encoding operation. There is reasonably good evidence for this from studies demonstrating that the associative links between trait descriptions depend on the processing schema. Lord

'It is worth noting here that we use the term "integrated" to refer to a set of cognitive operations that serve to link two or more representations in a consistent fashion. This is somewhat different from the use of the term in research stemming from Information Integration Theory (Anderson, 1974). 
(1980), for example, found that encoding information in the context of a "self" schema produces a more elaborated trace than encoding the same information in a different (i.e., nonself) context, such as a "Walter Cronkite" schema (see also Markus, 1980; Rogers, Kuiper, \& Kirker, 1977; for comparable encoding effects in respect to general semantic information see Craik \& Tulving, 1975). Hartwick (1979) even suggested that different links are activated when different encoding structures are employed because subjects evidenced more affective associations following instructions to form an opinion than following instructions to memorize.

In the present study, under integrative judgment, traits are encoded within a single schema, namely, they are associatively linked to each other via schematic knowledge. Under discrete judgments, however, traits tend to be encoded separately with few associative links. Since retrieval is an increasing function of the degree of association among the to-be-retrieved elements as well as the degree of associations between the latter and established knowledge (cf. Craik \& Tulving, 1975; Mandler, 1979), memory following integrative judgment should be superior to that following discrete judgment. In addition, inconsistencies are presumed to be reconciled during integration. If so, then whether the trait descriptions were originally consistent or inconsistent should not affect their accessibility once they have been integrated. Retrieval, thus, should be invariant with the consistency of the description under integrative judgment. Generally spcaking, however, consistent descriptions are inherently more configurable and, thus, more readily retrievable than inconsistent ones (e.g., Bower, Clark, Lesgold, \& Winzenz, 1969; Rothbart, Evans, \& Fulero, 1979). Recall that a discrete judgment is assumed to preserve the inconsistencies in a trait description. As a consequence, under discrete judgment consistent descriptions should be retrieved more readily than inconsistent ones.

\section{METHOD}

\section{Subjects}

Forty students from the University of Michigan introductory psychology classes served as subjects to satisfy a course requirement. They were assigned randomly to one of the two judgment conditions with the constraint that there be an equal number per condition.

\section{Apparatus}

Subjects were seated in individual booths, equipped with a 12 in. monitor, two movable buttons labeled "old" and "new," and a six-button response box (buttons labeled from 1 to 6). The "old" button was moved to the subject's dominant hand. Presentation of the stimuli was controlled by a PDP-11 computer system with responses and reaction times recorded under the program control. 


\section{Procedure}

Prior to the experiment proper, subjects underwent practice training in which they became familiar with the buttons, the screen, and the experimental procedure. Numbers were used as stimuli during this session.

Judgmental phase. The same eight sets of three traits from Anderson (1968) were presented to each subject in a different random order. The four consistent sets contained traits with similar affective values. The positive set had a mean "likability" value of 503 and the negative set a mean of 99 , with a maximum within-set difference of 100 on a 600 point scale. The four inconsistent sets contained traits with different affective values. Their mean "likability" value was 286 , with a minimum within-set difference of 300 points on this scale. ${ }^{2}$ In the integrative judgment condition subjects indicated that their opinion of a person described by each set on a scale ranging from (1) very unfavorable to (6) very favorable. In the discrete judgment condition subjects indicated whether the majority of the traits in each set could describe a human being only or nonhumans as well, on a scale ranging from "human only" (1) to "humans and nonhumans as well" (6).

Retrieval phase. Following the judgmental phase, subjects were presented with 64 probes (eight probes for each original set). Half of these probes were "old" (contained only traits that appeared in the same set during the judgmental phase), and the remaining were "new" (contained at least one trait that did not appear during the judgmental phase at all). Specifically, let (A B C) denote an original set (from the judgmental phase), and $\mathrm{N}$ denote a trait that did not appear in that phase. The "old" recognition probes were (A B). (A C), (B C), and (A B C). The "new" probes were chosen from the combinations ( $\mathrm{A} \mathrm{N}),(\mathrm{B} \mathrm{N}),(\mathrm{N} \mathrm{B}),(\mathrm{N} \mathrm{C}),(\mathrm{A} \mathrm{B} \mathrm{N}),(\mathrm{A} \mathrm{N} \mathrm{C})$, and (N C B).

For each original set two "new" probes contained only two traits, and the remaining two contained threc traits. In addition, for the consistent sets, two of the recognition probes contained a new trait that was evaluatively similar to the trait it replaced, and two "new" probes contained a new trait which was opposite in value. Probes were constructed so that each trait (old or new) was equally likely to be presented. Finally, the order in which probes were presented, as well as the particular "new" probes shown were determined randomly for each individual subject. Subjects responded by pressing a button labeled with "old" if the probe was a part of an original set, and with "new" otherwise.

\section{RESULTS AND DISCUSSION}

\section{Judgmental Phase}

The two judgment conditions were assumed to differ in respect to whether or not trait descriptions had to be integrated and inconsistencies among these descriptions resolved. Hence, it was expected that an inconsistent trait description would hinder processing under integrative judgment but not under discrete judgment. For each subject we computed the median response time. This was done separately for consistent and inconsistent trait sets. The means of these scores under the two judgment conditions are presented in Fig. 1. A two way repeated measure ANOVA (judgment type $\times$ consistency) was performed on these medians. Overall, responses to consistent sets were faster than those to inconsistent ones

${ }^{2}$ Traits which are evaluatively inconsistent may still be semantically consistent (e.g., Peabody, 1967). It has been demonstrated, however, that trait descriptions that are evaluatively inconsistent are most often seen as descriptively inconsistent (De Boack, 1978; Peabody, 1978; Rosenberg \& Olsham, 1970). 
$(F(1,38)=23.2, p<.01)$. The relationship between processing time and consistency, however, depended on the type of judgment, as indicated by the interaction between judgment type and consistency $(F(1$, $38)=24.9, p<.01$ ). Thus, there was essentially no difference between the encoding of consistent and inconsistent trait descriptions under discrete judgment, but a large difference under integrative judgment (see Fig. 1). Finally, there was no overall difference in response time between the two judgment conditions $(F<1)$.

\section{Retrieval Phase}

Recognition memory was sufficiently imperfect (mean proportion correct $=.71$ ) to raise the possibility of a tradeoff between accuracy and speed of response (Pachella, 1974). Recognition times, therefore, were not analyzed. For each subject we computed a biased-free sensitivity score, $A^{\prime}$, according to a procedure outlined in Grier (1971). This was done separately for consistent and inconsistent sets. These scores were then analyzed in a two-way repeated measures ANOVA (judgment type $\times$ consistency). ${ }^{3}$ The analysis indicated that subjects in the integrative judgment condition were more sensitive (i.e., performed more accurately in the recognition memory tasks after response biases were partialed out) than those in the discrete judgment condition $(F(1,38)=30.6, p<.01)$. More importantly, consistency tended to have different effects depending on the type of judgment. This is suggested by the marginally reliable interaction between consistency and type of judgment $(F(1,38)=3.19$, $p=.08$ ): Planned comparisons (Winer, 1971, p. 551) revealed that, as hypothesized, subjects under integrative judgment condition seemed to display the same high degree of sensitivity to consistent as well as to inconsistent sets $(t(18)=.08)$, whereas those under discrete judgment did not. In the latter condition subjects were more sensitive to consistent than to the inconsistent information $(t(18)=3.79, p<.05)$.

A comparison between recognition accuracy with similar and dissimilar "new" probes can shed additional light on the recognition process. In particular, if individuals store the gist of a trait description as indicated by its affective value, rather than an exact lexical representation, then it ought to be easier to reject affectively dissimilar "new" probes than affectively similar ones. To test this hypothesis we computed for each subject his or her accuracy rate (proportion correct) in recognizing the "new" probes to consistent sets. The computations were made separately for the similar and dissimilar probes.

${ }^{3}$ A three-way ANOVA (judgment $\times$ consistency $\times$ probe size) was conducted to explore whether the number of traits in the recognition probe affected recognition performance. Probes containing two traits produced greater sensitivity $(x=.79)$ than those containing three traits $(x=.74), F(1,34)=4.77, p<.05$. However, none of the interactions involving probe size approached acceptable levels of significance. 


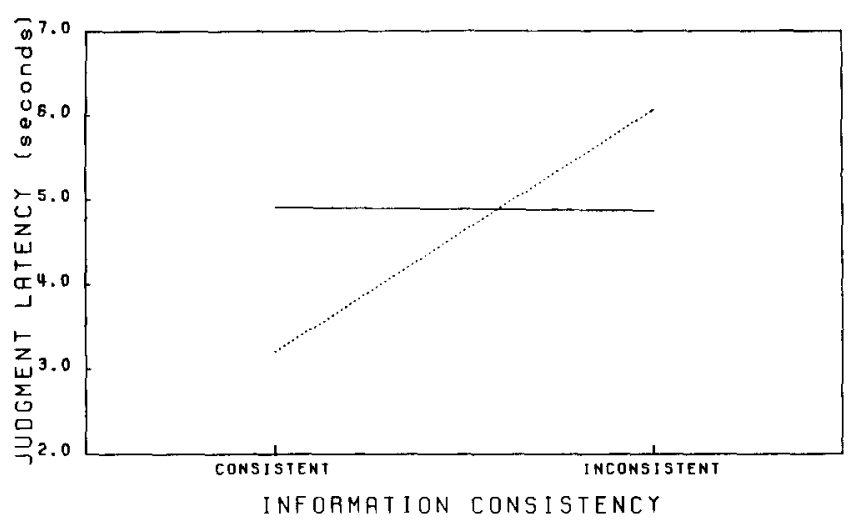

Fig. 1. Mean judgment time as a function of information consistency and processing task. (-) Discrete judgment: $(\cdots)$ integrative judgment.

A two-way repeated measures ANOVA (judgment type $\times$ probe type (similar vs dissimilar)) was performed on these accuracy rates. As in the $\mathrm{A}^{\prime}$ analysis, subjects under integrative judgment $(M=.80)$ were more accurate than those under discrete judgment $(M=.55), F(1,38)=17.0$, $p<.01$. More importantly, "new" probes that were affectively dissimilar to the description were rejected with greater accuracy $(M=.73)$ than ones that were affectively similar $(M=.62, F(1,38)=5.82, p<.05)$. The interaction between type of judgment and type of probe failed to reach significance $(F<1)$.

These findings suggest that when all the traits in a set have the same affective significance, the person can distinguish between similar or dissimilar "new" probes and the representation of the trait information whether or not this information has been integrated. It would appear, then, that subjects rely heavily on the more general (affective) meaning

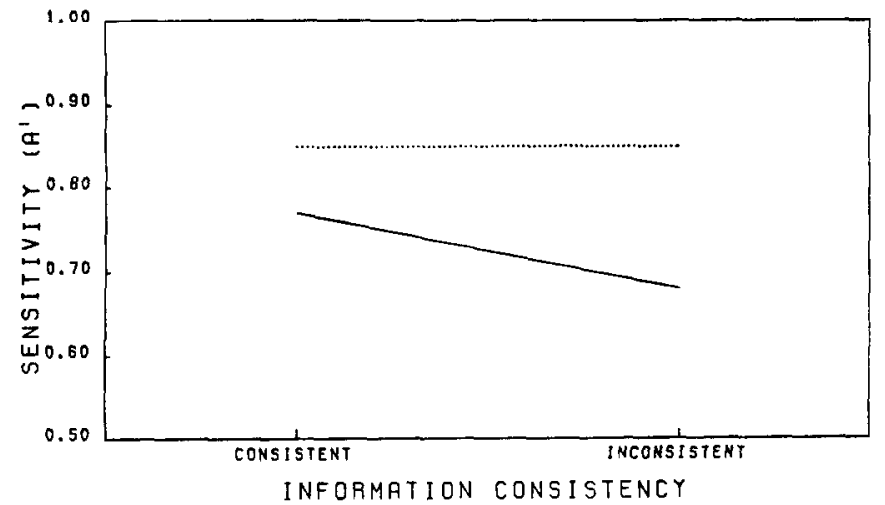

FIG. 2. Mean sensitivity $\left(A^{\prime}\right)$ as a function of information consistency and processing task. (-) Discrete judgment; $(\cdots)$ integrative judgment. 
of the traits in order to perform the recognition task. This leads to the speculation that individuals either retain little of the lexical features of a trait or they store these separate from, and in less accessible form than, the affective features (cf. Anderson \& Hubert, 1963). These results, however, are merely suggestive in this respect. A more definitive test would require systematic comparison of lexical and affective features, which was not possible in our study. Nevertheless, the same phenomenon, retention of the gist rather than the lexical information in a message. has been documented repeatedly with prose (e.g., Bransford \& Franks, 1971).

In related studies, Bower and Karlin (1974) and Winograd (1976) found better recognition memory for faces when the encoding task required integration of facial cues, that is, when the faces were interpreted within a relatively rich schema, than when integration was not required (see also Lord, 1980; Markus, 1980). In addition, of course, there are wellknown experiments that obtain comparable memory effects for semantic information in general (e.g., Craik \& Tulving, 1975) and for person descriptions in particular (e.g., Hastie \& Kumar, 1979). These authors explained their results primarily in terms of levels of processing theory (Craik \& Lockhart, 1972).

Although the above findings parallel our own, the level-of-processing explanation seems not to apply in the present case. Not only were there no overall differences in processing times between our two judgment conditions that could suggest a difference in processing depth, but more importantly, the interaction between consistency and judgment type in respect to processing time was the mirror image of the comparable interaction in respect to retrieval. That is to say, during the judgmental phase there was no difference due to consistency for the discrete judgment group, but a large difference for the integrative judgment group; whereas during retrieval there was a clear difference in sensitivity due to consistency for the former, but no difference for the latter. These effects, however, do follow from our assumption about the distinction between integrative judgments and discrete judgments, namely, that even though the two are comparable in respect to level of processing-both involve semantic elaboration of the trait descriptions-they differ in respect to whether or not the elaborated descriptions needed to be integrated.

Our initial research on opinion formation and social judgment (Burnstein \& Schul, 1982) suggested that elaborative encoding and integration were functionally distinct processes. This was based on their differential sensitivity to changes in the consistency of a trait description so that an inconsistent description was observed to have a larger impact on processing time during integration than during elaborative encoding. The present findings not only demonstrate this effect on processing in 
a more clear-cut fashion, but more importantly, indicate that it extends also to the representation of a trait description.

\section{REFERENCES}

Anderson, N. H. Likableness ratings of 555 personality-trait words. Journal of Personality and Social Psychology, 1968, 9, 272-279.

Anderson, N. H. Information integration theory: A brief survey. In D. H. Krantz, R. C. Atkinson, R. D. Luce, \& P. Suppes (Eds.), Contemporary development in mathematical psychology (Vol. 2). San Francisco: Freeman, 1974.

Anderson. N. H., \& Hubert, S. Effect of concomitant verbal recall on order effects in personality impression formation. Journal of Verbal Learning and Verbal Behavior, 1963, 2, 379--391.

Bower, G., \& Karlin, M. Depth of processing pictures of faces and recognition memory. Jourtal of Experimental Psychology, 1974, 103, 751-757.

Bower, G. H., Clark, M. C., Lesgold, A. M., \& Winzenz, D. Hierarchical retrieval schemes in recall of categorical word lists. Journal of Verbal Learning and Verbal Behavior, $1969,8,323-343$.

Bransford, J. D., \& Franks, J. J. The abstraction of linguistic ideas. Cognitive Psychology, $1971,2,331-350$.

Burnstein, E., \& Schul, Y. The informational basis of social judgments: The operations in forming an impression of another person. Journal of Experimental Social Psychology, 1982, 18, 217-234.

Craik, F. I. M., \& Lockhart, R. S. Levels of processing: A framework for memory research. Journal of Verbal Learning and Verbal Behavior, 1972, 11, 671-686.

Craik, F. I. M., \& Tulving. E. Depth of processing and the retention of words in episodic memory. Journal of Experimental Psychology, 1975, 104, 268-294.

De Boack, P. On the evaluative factor in the trait scales of Peabody's study of trait inferences. Journal of Personality and Social Psychology, 1976, 36, 619-621.

Grier, J. B. Nonparametric indexes for sensitivity and bias: Computing formulas. Psychological Bulletin, 1971, 75, 424-429.

Hartwick, J. Memory for trait information: A signal detection analysis. Journal of Experimental Social Psychology, 1979, 15, 533-552.

Hastie, R., \& Kumar, P. A. Person memory: Personality traits as organizing principles in memory for behavior. Journal of Personality and Social Psychology, 1979, 37, $25-38$.

Holmes, V. M. Accessing ambiguous words during sentence comprehension. Quarterly Journal of Experimental Psychology, 1979, 31, 569-589.

Lord, C. G. Schemas and images as memory aids: Two modes of processing social information. Journal of Personality and Social Psychology, 1980, 38, 257-269.

Mandler, G. Organization and repetition: Organizational principles with special reference to rote learning. In L. G. Nilsson (Ed.), Perspectives in memory research. Hillsdale, N.J.: Erlbaum, 1979.

Markus, H. The self in thought and memory. In D. Wegner \& R. Vallacher (Eds.), The self in social psychology. Oxford: Oxford Univ. Press, 1980.

Pachella, R. G. The interpretation of reaction time in information-processing research. In B. Kantowitz (Ed.), Human information processing: Tutorials in performance and cognition. Hillsdale, N.J.: Erlbaum, 1974.

Peabody, D. Trait inferences: Evaluative and descriptive aspects. Journal of Personality and Social Psychology Monograph, 1967, 7(4, Whole No. 644).

Peabody, D. In search of an evaluative factor: Comments on De Boack. Journal of Personality and Social Psychology, 1978. 36, 622-627. 
Rogers, T.. Kuiper, R., \& Kirker, W. Self reference and encoding of personal information. Journal of Personality and Social Psychology, 1977, 35, 677-688.

Rosenberg, S. \& Olshan, K. Evaluative and descriptive aspects in personality perception. Journal of Personality and Social Psychology, 1970. 16, 619-626.

Rothbart, M., Evans, M., \& Fulero, S. Recall for confirming events: Memory processes and maintenance of social stereotypes. Journal of Experimental Social Psychology, $1979,15,343-355$.

Smith. E.. Shoben, E., \& Rips. L. Structure and process in semantic memory: A featural model for semantic decisions. Psychological Review, 1979, 81, 214-241.

Winer, B. J. Statistical principles in experimenter design. New York: McGraw-Hill, 1971.

Winograd, E. Recognition memory for faces following nine different judgments. Bulletin of the Psychonomic Society, 1976, 8, 419-421. 